\title{
An Enhencment Medical Image Compression Algorithm Based on Neural Network
}

\author{
Manel Dridi \\ Electronics and Micro-Electronics Laboratory \\ (E. $\mu$. E. L), University of Monastir - Tunisia \\ National Engineering School of Monastir, University of \\ Monastir - Tunisia \\ Mohamed Ali Hajjaji \\ Electronics and Micro-Electronics Laboratory \\ (E. $\mu$. E. L), University of Monastir - Tunisia \\ Higher Institute of Applied Sciences and Technology of \\ Kasserine, University of Kairouan
}

\author{
Belgacem Bouallegue \\ Electronics and Micro-Electronics Laboratory \\ (E. $\mu$. E. L), University of Monastir - Tunisia
}

Abdellatif Mtibaa

Electronics and Micro-Electronics Laboratory

(E. $\mu$. E. L), University of Monastir - Tunisia

National Engineering School of Monastir, University of

Monastir - Tunisia

\begin{abstract}
The main objective of medical image compression is to attain the best possible fidelity for an available communication and storage [6], in order to preserve the information contained in the image and does not have an error when they are processing it. In this work, we propose a medical image compression algorithm based on Artificial Neural Network (ANN). It is a simple algorithm which preserves all the image data. Experimental results performed at 8 bits/pixels and 12bits/pixels medical images show the performances and the efficiency of the proposed method. To determine the 'acceptability' of image compression we have used different criteria such as maximum absolute error (MAE), universal image quality (UIQ), correlation and peak signal to noise ratio (PSNR).
\end{abstract}

Keywords_Artificial Neural Network; medical image;
compression; DICOM; PSNR;CR

\section{INTRODUCTION}

DICOM (Digital Imaging and Communications in Medicine) image is composed of two files [15]: header files and image pixel data. Therefore, the treatment of digital medical images as textual data, such as computed tomography (CT) and the magnetic resonance images (MRIs) required an extensive amount of hard disk space, also a high transmission time needed to store and to transmit because of the large file size [9]. One of the solutions is to compress medical images before its transmission and storage, then decompress it at the receiver for use. At present, the main steps of image compression are: pixels transform, quantization and entropy coding [11]. In the last years, compression methods have attracted the interest of many researchers all around the world; many compression methods have been proposed. Among the already proposed methods such as JPEG2000, and JPEG cannot be satisfied due to the long execution time, complexity of algorithms or/and lower compression ratio.

As flown we present some researchers work. In [5] M.ElZorkany has proposed a new image compression approach which combines NN and DCT. In [9] W.K.Yeo et al. have presented their medical image compressed algorithm, which is based on Hebbian process and quantization of the extracted components. In [10] S.manimurugan et al. proposed a cryptocompression medical image using the block pixel sort scheme. In [18] A.Younus et al. have proposed a hybrid medical image compression technique based on Discrete Cosines Transform (DCT) and Lapped Biorthogonal Transform (LBT). In [7] S.kuamo et al. present an experimental study of some image compression methods and propose a new hybrid method based on a neural network.

This paper is organized as follows, in the second section, we define neural network and give its different features. In the third section we present the proposed scheme. In the fourth section, we present some treated examples and tests followed by some comparison with respect to other works. Finally, we conclude this paper.

\section{ARTIFICIAL NEURAL NETWORK (ANN)}

In this section, we present a brief introduction of Artificial Neural Network (Fig.1), which is inspired from human brains. It's a data modeling tool able to capture and represent a complex input/output relationship. Because of the specified characteristic for ANN such as high degree of interconnection, nonlinear mapping, massive parallel structure and selforganization, we can estimate that ANN can resolve some compression problems [12].

The ANN parameters are : the number of training iterations and hidden neurons , the input layer units for each hidden layer unit $(\mathrm{N})$ and the range of synaptic weight values (MINMAX) $\{-\mathrm{L} \ldots+\mathrm{L}\}$. The main steps for ANN are:

\section{A. Initiation}

Put all weights and activation thresholds of the network to random values uniformly distributed in a small range. This initialization is done for one neuron. Set the value of the learning rate to a small positive value. 


\section{B. Activation}

The activation function in ANNs algorithm is a weighted sum: the sum of the inputs $X$ multiplied by their respective weights $\mathrm{w}_{\mathrm{ji}}$ ):

$$
X j=f\left(\sum_{i=1}^{N} w_{i j} \times x_{i}\right) \text { With } 1 \leq j \leq M
$$

Activate the neural network using input signals and desired output.

Calculate the output signals of the neurons of the successive layers and the first hidden layer to the output layer (see eq 1).

$$
\text { Where } f(x)=\frac{1}{1+e^{-x}}
$$

\section{Training weights:}

Update the weights of the network by propagating the error in the opposite direction

$$
\mathrm{w}_{\mathrm{i}}(\mathrm{p}+1)=\mathrm{w}_{\mathrm{i}}+\Delta \mathrm{w}_{\mathrm{i}}
$$

Calculate the error gradient for the hidden layers (reverse order) and for the output layer.

Calculate the weight corrections for each layer and update the weights.

\section{Iteration}

Increment of p, return to step 2 and repeat the process while the error is reached

1) Set a random weight were used to start. The weights were usually between 0.01 and .99 .

2) Calculate each output layer

3) Calculate errors: the error of the neuron $k$ is the difference between the value of the desired output and the actual value of the output neuron $\mathrm{k}$

$$
e_{k}(p)=y_{d, k}-y_{k}
$$

4) Compute delta errors

$$
\Delta \mathrm{w}_{\mathrm{jk}}(\mathrm{p})=\alpha \times \mathrm{y}_{\mathrm{j}}(\mathrm{p}) \times \delta_{\mathrm{k}}(\mathrm{p})
$$

Where $\delta_{k}(p)$ is the neuron error gradient $\mathrm{k}$ at $\mathrm{p}$ iteration.

a) The error Gradient for output layer neurons

$$
\begin{array}{r}
\delta_{\mathrm{k}}(\mathrm{p})=\mathrm{f}^{\prime}\left[\mathrm{X}_{\mathrm{k}}(\mathrm{p})\right] \times \mathrm{e}_{\mathrm{k}}(\mathrm{p}) \\
=\mathrm{y}_{\mathrm{k}}(\mathrm{p}) \times\left[1-\mathrm{y}_{\mathrm{k}}(\mathrm{p})\right] \times \mathrm{e}_{\mathrm{k}}(\mathrm{p})
\end{array}
$$

The second part is determined by the derivative of the sigmoid function applied to the input net of the neuron $\mathrm{k}$ at $\mathrm{p}$ iteration

b) The error Gradient for hidden layer neurons

$\delta_{j}(p)=y_{j}(p) \times\left[1-y_{j}(p)\right] \sum_{k=1}^{l} \delta_{k}(p) w_{j, k}(p)$

\section{COMPRESSION ALGORITHM: THE PROPOSED ALGORITHM}

In this section, we present the proposed method in detail (Fig 2). There are many different types from ANN [5] in our work; we have used Multilayer Perceptron (MLP) with three layer input layer, one hidden layer and an output layer as shown in Fig.1 above.

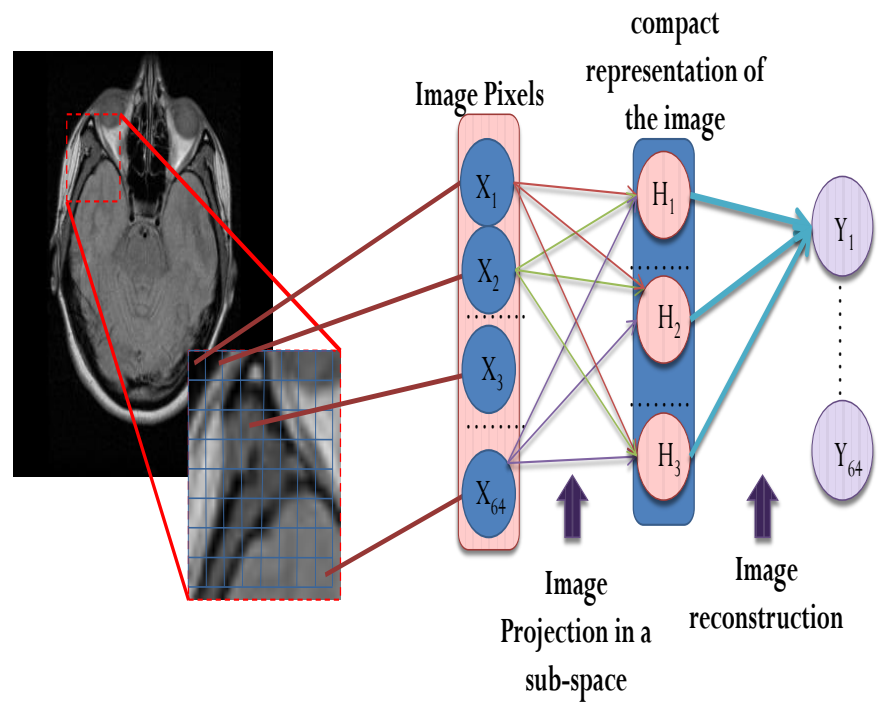

Fig. 1. Architecture of neural network

First off, the entire pretreatment step: the image, I, pixels is converted to double data type then normalized ( $\mathrm{I}=\mathrm{I} / 4000)$. Secondly, the image is divided into square blocks of $\mathrm{n}$ by $\mathrm{n}$ size in order to reduce execution time. Then each of these blocks are transformed into a vector of $\mathrm{N}$-dimensional. The input vectors of attributes, $X_{N}$, to the input layer can be written as $\mathrm{X}_{\mathrm{K}}=\left\{\mathrm{x}_{1} \ldots \ldots \mathrm{xi} \ldots \ldots \mathrm{x}_{\mathrm{N}}\right\}$ where $\mathrm{K}=(1,2, \ldots, \mathrm{K}), \mathrm{K}$ is the total vector number. Thirdly, for the training step in which the data of every vector are entered in the network as an input data iteratively: In each iteration, the network weights are updated according to equation (2), and the residues error obtained as the difference between the actual and the predicted calculated output. The error is used to adjust the weights. The training continues until the predictive error is reached. The allowable error is equal $1 \mathrm{e}^{-10}$. Finally, the corresponding encoding from the obtained hidden layer is saved with the output weight. To get the decompressed image, we use the appropriate compressed image data and extracted weights to train the neural network. The appropriate compressed image is entered in the output layer block by block and the output data are computed in order to recreate the original image.

Where 1 is the number of neurons in the next layer 


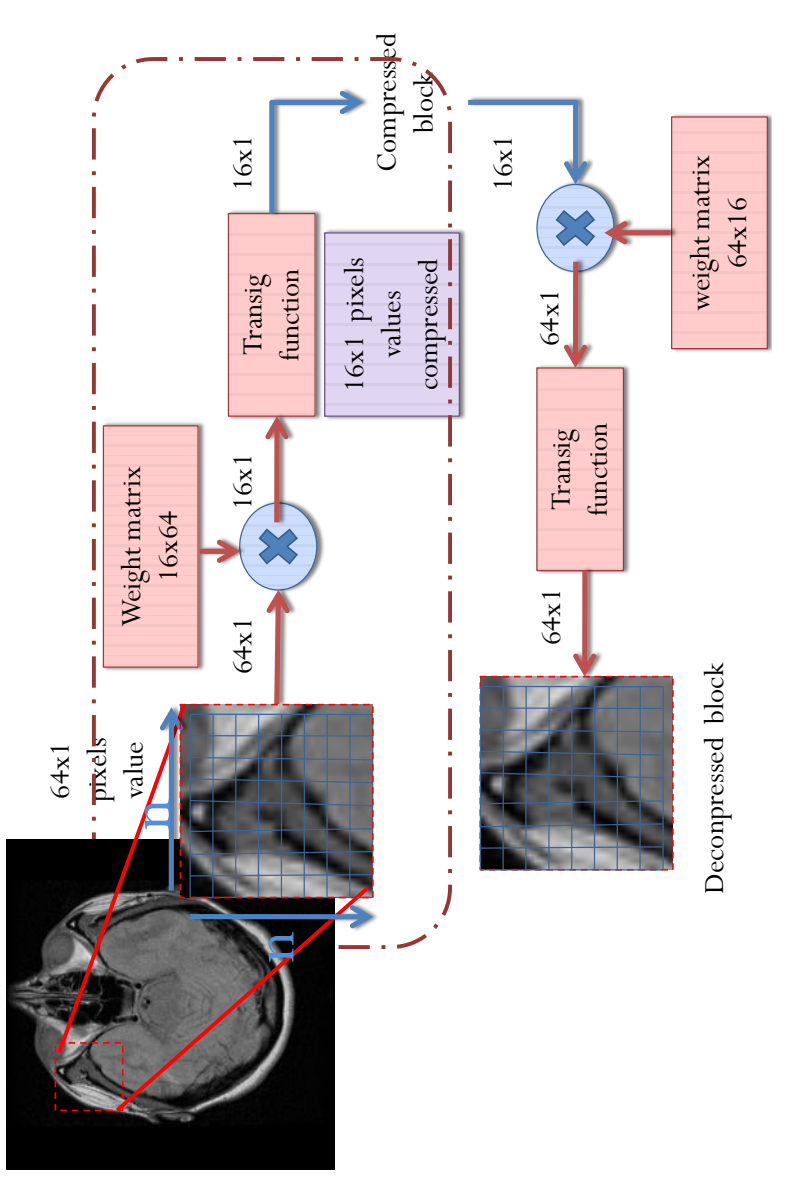

Fig. 2. The proposed process

\section{The algorithm}

Step 1: [Start] Generate a random weight and divided the image in sub blocks

$$
\left(\mathrm{w}_{\mathrm{ij}}=[0.5-\operatorname{rand}(\mathrm{nbc} 2, \mathrm{nbc} 1)]\right.
$$

Step 2: calculate hidden and output layer

$$
\begin{gathered}
\mathrm{s} 1 \mathrm{j}=\sum_{i=0}^{n} w_{j}(i) \times \operatorname{imp}(i) \\
\mathrm{s} 2 \mathrm{k}=\sum_{j=0}^{n} w_{k}(j) \times \mathrm{H}(j) \\
\mathrm{H}=[2 . /(1+\exp (-\mathrm{s} 1))]-1 ; \\
\text { out }=[2 . /(1+\exp (-\mathrm{s} 2))]-1 ;
\end{gathered}
$$

Step 3: Create a new weight by repeating the following steps until we get a perfect quality of image.

$\mathrm{e}(\mathrm{k})=\mathrm{d}(\mathrm{k})$-out $(\mathrm{k})$;

$$
\begin{gathered}
\mathrm{E}=\mathrm{E}+\left(1 / 2^{*}\left((\mathrm{e}(\mathrm{k}))^{\wedge} 2\right)\right) ; \\
\operatorname{derfs} 2=(1-\mathrm{out}) *^{*}(1+\mathrm{out}) ; \\
\operatorname{delta} 1=\mathrm{e} \cdot{ }^{*} \operatorname{derfs} 2 ; \\
\operatorname{gradES}(\mathrm{j}, \mathrm{k})=\mathrm{mu}^{*} \operatorname{delta} 1(\mathrm{k})^{*} \mathrm{H}(\mathrm{j}) ; \\
\mathrm{w} 1=\mathrm{w} 1+\operatorname{gradES} ; \\
\operatorname{gradEC}(\mathrm{i}, \mathrm{j})=\mathrm{mu}{ }^{*} \operatorname{delta} 2(\mathrm{j}) * \operatorname{mimp}(\mathrm{i}) ; \\
\mathrm{w}=\mathrm{w}+\operatorname{gradEC} ;
\end{gathered}
$$
run.

Step 4: Use new generated weight for a further algorithm

Step 5: [Test] if the end condition is satisfied, stop, and return the best solution
Step 6: Go to step 2.

According to the Neural network the process of compression is presented by eq (8) for encoding:

$$
h_{j}=f\left(\sum_{i=1}^{N} w_{i j} \times x_{i}\right) \text { with } 1 \leq j \leq M
$$

Where $f(x)$ is a sigmoid activation function:

$$
f(x)=\frac{2}{1+e^{-x}}
$$

and by eq (9) for decoding

$$
y i=f\left(\sum_{j=1}^{M} w_{i j} \times h_{j}\right)-1
$$

with $1 \leq \mathrm{i} \leq \mathrm{N}$

Where $\mathrm{y}_{\mathrm{i}} \epsilon[0,1]$ and presents the normalized pixel values. We used normalized pixel values because the neural network can perform more efficiently when their inputs and outputs belong to the interval $[0,1]$.

\section{EXPERIMENTAL RESULTS}

The proposed approach for the compressed medical image is implemented in Matlab using a personal computer with an intel dual core $2.2 \mathrm{GHz}$ processor, windows7.

In order to evaluate the performances of our approach, we were considered some indicators which are the most used in the research area:

\section{A. Definition}

\section{- $\mathbf{C R}$}

$\mathrm{CR}$ is the ratio between the uncompressed and compressed image size

\section{- MSE}

$$
\text { Compressionratio }=\frac{\text { Original image size }}{\text { Compressed image size }}
$$

Mean Squared Error is the cumulative squared error between the compressed and the original image. The large value of MSE means that image is poor quality. It is defined as follows:

$$
\mathrm{MSE}=\frac{1}{\mathrm{~W} \times \mathrm{L}} \sum_{\mathrm{i}=1}^{\mathrm{W}} \sum_{\mathrm{j}=1}^{\mathrm{H}}[\mathrm{P}(\mathrm{i}, \mathrm{j})-\mathrm{C}(\mathrm{i}, \mathrm{j})
$$

- MAE (absolute error)

The MAE between two images is defined according to two images, $\mathrm{P}(\mathrm{i}, \mathrm{j})$ and $\mathrm{C}(\mathrm{i}, \mathrm{j})$, which correspond to the plain-image and the decompressed image. It's defined by equation (12)

$$
\mathrm{MAE}=\frac{1}{\mathrm{~W} \times \mathrm{L}} \sum_{\mathrm{i}=1}^{\mathrm{W}} \sum_{\mathrm{j}=1}^{\mathrm{H}}\left|\mathrm{P}(\mathrm{i}, \mathrm{j})_{-} \mathrm{C}(\mathrm{i}, \mathrm{j})\right| \times 100 \%
$$

\section{- PSNR [9]}

It means the Peak Signal to Noise Ratio which can be used to evaluate a compression scheme. It's defined as follow by equation (14) in which $b$ is the number of bit coding.

$$
\operatorname{PSNR}=10 \times \log _{10}\left[\frac{\mathrm{L} \times \mathrm{W} \times \max (\mathrm{P})^{2}}{\sum_{\mathrm{i}=1}^{\mathrm{L}} \sum_{\mathrm{j}=1}^{\mathrm{W}}(\mathrm{P}(\mathrm{i}, \mathrm{j})-\mathrm{C}(\mathrm{i}, \mathrm{j}))^{2}}\right]
$$


Typically, the values of PSNR, for lossy compression of an image, are between 30 and $50 \mathrm{~dB}$ and when the PSNR is greater than $40 \mathrm{~dB}$, then the two images are indistinguishable.

- Correlation analysis

In an ordinary image, each pixel is highly correlated with its adjacent pixel. However, the test of the adjacent pixels correlations in the decompressed image determines the superior confusion and diffusion characteristics [8]. The correlation of the adjacent pixels is calculated with the use of Eq. (15), (16), (17) and (18).

$$
\begin{aligned}
& \mathrm{E}(\mathrm{x})=\frac{1}{\mathrm{~N}} \sum_{\mathrm{i}=1}^{\mathrm{N}} \mathrm{x}_{\mathrm{i}} ; \\
& \mathrm{D}(\mathrm{x})=\frac{1}{\mathrm{~N}} \sum_{\mathrm{i}=1}^{\mathrm{N}}\left(\mathrm{x}_{\mathrm{i}}-\mathrm{E}\left(\mathrm{x}_{\mathrm{i}}\right)\right)^{2} \\
& \operatorname{cov}(\mathrm{x}, \mathrm{y})=\frac{1}{\mathrm{~N}} \sum_{\mathrm{i}=1}^{\mathrm{N}}\left(\mathrm{x}_{\mathrm{i}}-\mathrm{E}(\mathrm{x})\right)\left(\mathrm{y}_{\mathrm{i}}-\mathrm{E}(\mathrm{y})\right) \\
& \mathrm{r}_{\mathrm{xy}}=\frac{\operatorname{cov}(\mathrm{x}, \mathrm{y})}{\sqrt{\mathrm{D}(\mathrm{x})} \sqrt{\mathrm{D}(\mathrm{y})}},
\end{aligned}
$$

where $x$ and $y$ are grayscale values of two adjacent pixels in the image. $\mathrm{E}(\mathrm{x})$ is the expectation of $\mathrm{x}, \mathrm{D}(\mathrm{x})$ is the estimation of the variance of $\mathrm{x}$ and $\operatorname{cov}(\mathrm{x}, \mathrm{y})$ is the estimation of the covariance between $\mathrm{x}$ and $\mathrm{y}$.

\section{B. Results and interpretation}

\section{1) Results}

In this part, we have given different performance analysis of the proposed process. By using the formula presented in the previous part, the evaluation of the reconstructed image was performed.

Table 1, Table 2 and Table 3 represent the PSNR results, Universal Image Quality Index, SNR and correlation of some medicals images. These results illustrate that, if the input and the hidden layer numbers decrease the PSNR and Universal Image Quality Index will also increase. As shown in a tab, it was found that the PSNR was between $34 \mathrm{~dB}$ and $50 \mathrm{~dB}$.

We remark from correlation values results that there is an important relation between the pixels of the original image and the decompressed one.

2) The influence of the size of sub-block

In this section, we show the influence of the number of the input layer and the number of the hidden layer on the quality of the obtained image after decompression. Presence of hidden neurons enhances the flexibility of the system and increases power processing, but if the number of hidden neurons is taken too small, then robustness of the system can reduce due to improper fitting of input data. Therefore, in order to have a good result, we don't use few neurons in the hidden layers.

If we observe the graph (Fig 3, Fig 4 and Fig5) and the Fig 6 presented above, we can conclude that the use of 64 inputs for the input layer provides a more reliable quality image than the use of 36 inputs for the input layer.

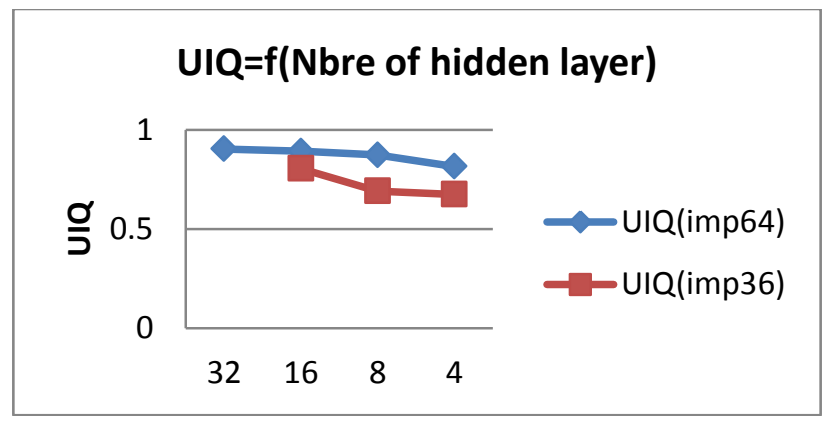

Fig. 3. universal image quality

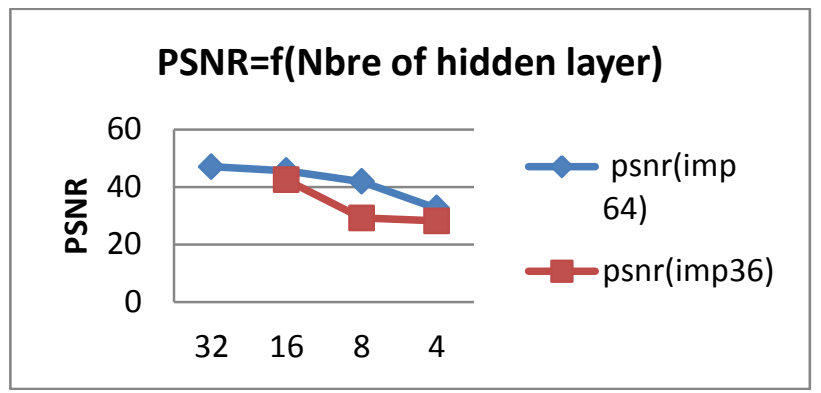

Fig. 4. PSNR (db)

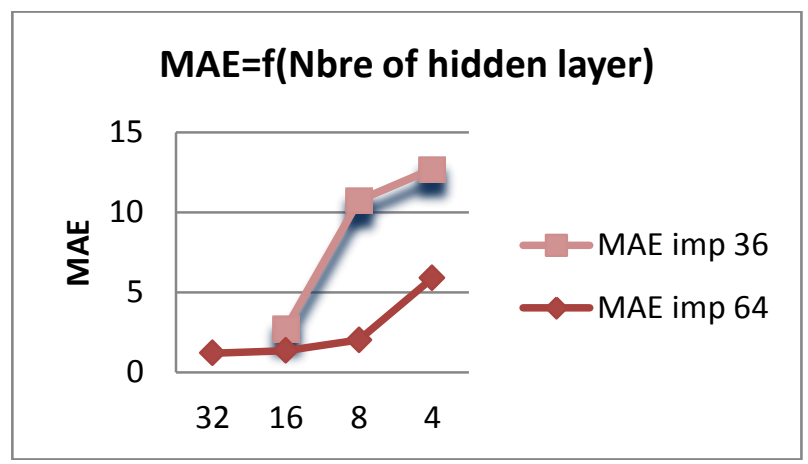

Fig. 5. MAE

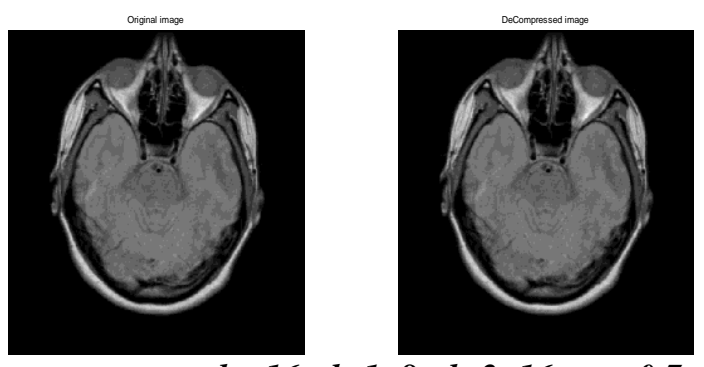

a. $l x=16 ; n b c 1=8 ; n b c 2=16 ; m u=0.7$;
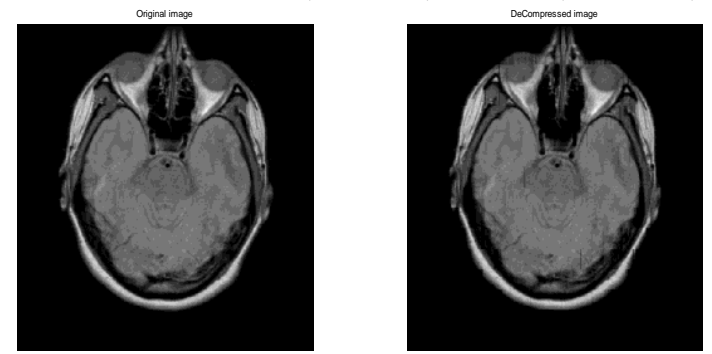

b. $L x=64 ; n b c 1=32 ; n b c 2=64, m u=0.7$ 

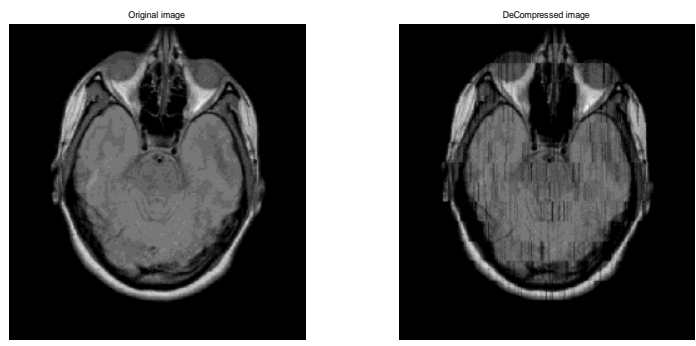

c. $l x=16 ; n b c 1=8 ; n b c 2=16 ; m u=0.7 ;$

Fig. 6. sub-blocks size influence

We have performed several simulations to find the best parameters in order to have a better decompressed image quality. As seen in previous figure5, we note that for $\mathrm{mu}=0.7$ the resulting image is acceptable. But this result is just for some images, but for another one we should find the parameter mu which gives a good result.

TABLE I. NUMBER OF INPUT $=64$, NUMBER OF HIDDEN LAYER $=32$ NUMBER OF OUTPUT $=64 ; \mathrm{MU}=0.6$ EXPECT FOR $2 \mathrm{MU}=0.2$

\begin{tabular}{|l|l|l|l|l|}
\hline & PSNR(db) & UIQ & SNR & $\begin{array}{l}\text { Correlation of } \\
\text { decompressed } \\
\text { image }\end{array}$ \\
\hline 1 & 47.2245 & 0.81371 & 35.7104 & 0.99954 \\
\hline 2 & 45.0224 & 0.68115 & 33.0231 & 0.9997 \\
\hline 3 & 44.3494 & 0.77337 & 33.8874 & 0.99951 \\
\hline 4 & 45.5447 & 0.78747 & 34.537 & 0.99952 \\
\hline 5 & 40.7745 & 0.78747 & 28.7745 & 0.99952 \\
\hline 6 & 42.9413 & 0.38702 & 26.2487 & 0.99747 \\
\hline 7 & 48.5782 & 0.69393 & 34.0252 & 0.99937 \\
\hline 8 & 50.2165 & 0.67070 & 35.402 & 0.99965 \\
\hline
\end{tabular}

TABLE II. NBRE OF INPUT $=64$, NBRE OF HIDEN LAYER $=16$, NBRE OF OUTPUT $=64 ; \mathrm{MU}=0.6$

\begin{tabular}{|l|l|l|l|l|}
\hline & PSNR $(\mathrm{dB})$ & UIQ & SNR & $\begin{array}{l}\text { Correlation of } \\
\text { decompressed } \\
\text { image }\end{array}$ \\
\hline 1 & 45.7308 & 0.89591 & 34.2158 & 0.99974 \\
\hline 2 & 46.0224 & 0.77833 & 34.0232 & 0.99982 \\
\hline 3 & 43.311 & 0.84740 & 32.8491 & 0.99966 \\
\hline 4 & 44.1974 & 0.86226 & 33.1964 & 0.99970 \\
\hline 5 & 37.363 & 0.99857 & 25.4508 & 0.99857 \\
\hline 6 & 39.5329 & 0.42105 & 23.8405 & 0.99816 \\
\hline 7 & 44.6791 & 0.83524 & 30.1262 & 0.99961 \\
\hline 8 & 48.1061 & 0.76283 & 33.2916 & 0.99975 \\
\hline
\end{tabular}

TABLE III. NBRE OF INPUT $=36$, NBRE OF HIDEN LAYER $=18$, NBRE OF OUTPUT $=36 ; \mathrm{MU}=0.6$

\begin{tabular}{|l|l|l|l|l|}
\hline & PSNR(dB) & UIQ & SNR & $\begin{array}{l}\text { Correlation of } \\
\text { decompressed } \\
\text { image }\end{array}$ \\
\hline 1 & 42.9718 & 0.80863 & 31.4568 & 0.99949 \\
\hline 2 & 43.9466 & 0.64007 & 31.9474 & 0.99974 \\
\hline 3 & 41.5185 & 0.76174 & 31.9474 & 0.99931 \\
\hline 4 & 41.8933 & 0.78607 & 30.8923 & 0.99949 \\
\hline 5 & 34.1588 & 0.50070 & 22.2459 & 0.99699 \\
\hline 6 & 38.05 & 0.37824 & 22.3635 & 0.99739 \\
\hline 7 & 42.6312 & 0.70103 & 28.0783 & 0.99938 \\
\hline 8 & 46.4447 & 0.66797 & 31.6303 & 0.99965 \\
\hline
\end{tabular}

\section{COMPARISON BETWEEN OTHER COMPRESSION METHODS}

In order to show the efficiency of the proposed process, we have compared it firstly with JPEG compression and with wavelet compression secondly. This comparison is carried out by calculating the compression ratio (CR) and peak signal to noise ratio (PSNR) for medical image compressed by JPEG, then by comparing the image resulting using the proposed algorithm with the image resulting using other proposed method.

As seen in Table 4, we can conclude that our proposed process gives a good result. In fact, our result is better than the result in 13 and 14 for $C R$ value.

TABLE IV. COMPARISON WITH OTHER WORKS

\begin{tabular}{|l|l|l|}
\hline & CR & PSNR(db) \\
\hline our & 7,44 & 32,08 \\
\hline 13 & Max $=3,05$ & 23.9 \\
\hline 14 & 5.387 & 42.499 \\
\hline
\end{tabular}

and as seen as the obtained image in [17] (Fig. 8.) we remark that the original image and the compressed one haven't the same psycho-visual characteristic. Contrariwise, we obtained the image (Fig.7) has the same psycho-visual characteristic. Since, we are working with medical images that may cause some problems.
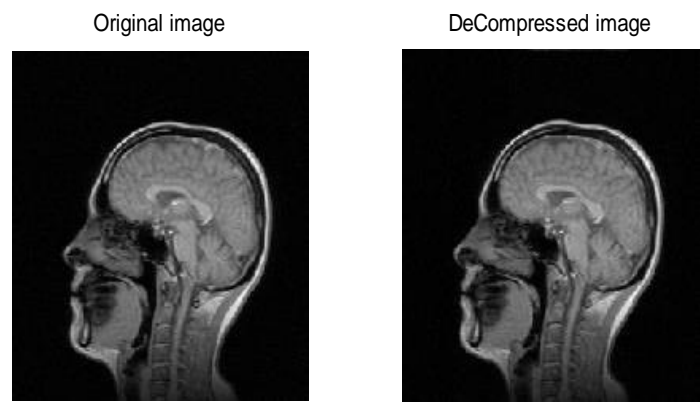

Fig. 7. The result image obtained by our proposed algorithm

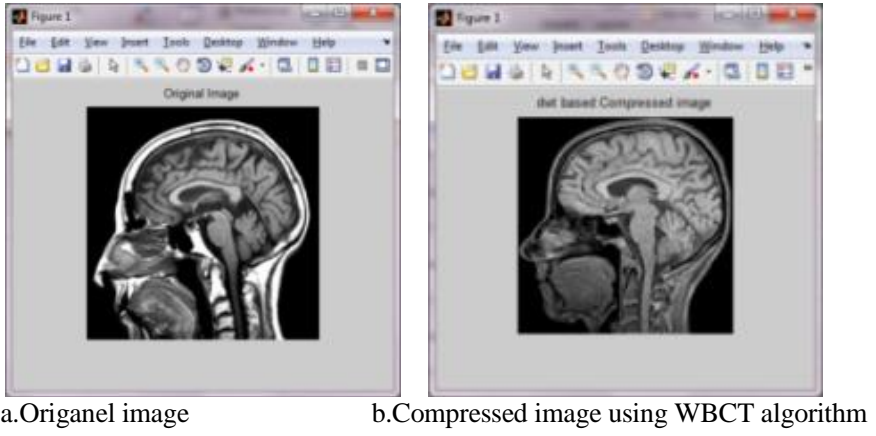




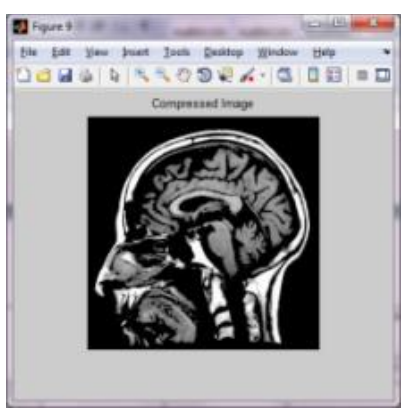

c. Compressed image using WDT

Fig. 8. The result image obtained in the related work [16]

Compared to the other works, our proposed scheme presents a compromise between the quality of the obtained image and the compression ratio. In addition, the proposed algorithm is more easily adapted than the other algorithm such as JPEG and JPEG2000.

\section{CONCLUSION}

In this work, we have proposed a compression method for medical image which preserve the quality of the original image. This method is based on neuronal network with some modification in the back-propagation usual algorithm. We have shown the efficiency and the performance of the proposed scheme by carrying out many measurements for differential analysis such, the correlation, the PSNR and the Universal Image Quality Index. The simplicity of the algorithm makes real-time implementation easy therefore in the future work we will implement this algorithm on FPGA.

\section{REFERENCES}

[1] Y. Shantikumar Singh, B. Pushpa Devi, Kh.Manglem Singh, Image Compression using Multilayer Feed Forward Artificial Network with conjugate Gradient ,World Congress on Information and Communication Technologies2012 IEEE.

[2] P.V Rao, S.Madhusudana, N.SS and KusumaKeerthi, Image Compression using Artificial Neural Networks, Second International Conference on Machine Learning and Computing, 2010.

[3] D.Bouslimi, G. Coatrieux, Christian Rouxa, A joint encryption/watermarking algorithm for verifying the reliability of medical images: Application to echographic images, computer methods and programs in (2012) pp47-54.

[4] U.Seiffert, ANNIE-Artificial Neural Network-based Image encoder, Neurocomputing 125(2014), pp:229-235.
[5] M.ElZorkany, A hybrid image compression technique using neural network and vector Quantization with DCT, Image Processing and Communications Challenges 5, Advance in Intelligent Systems and Computing 233,Springer International Publishing Switzerland, 2014.

[6] A.Saffor, A.R.Ramli and Kwan-Hoong Ng, A Comparative Study of Image Compression Between JPEG and WAVELET, Vol. 14 No. 1, June 2001, pp. 39-45.

[7] S.Kouano and C.Tangha, Image Compression with Artificial Neural Networks, Springer-Verlag Berlin Heidelberg 2013. Int. joint conf. CISIS'12-SOCO'12.AISC 189, 2012, pp: 515-524.

[8] Chong Fu a,n, Wei-hongMeng b, Yong-fengZhan b, Zhi-liangZhu c, FrancisC.M.Lau d, Chi K.Tse d, Hong-fengMae ,An efficient and secure medical image protection scheme based on chaotic maps, Science Direct journal ,Computers inBiology and Medicine,2013.

[9] W.K Yeo, D.F.W.Yap,D.P.Andito, MK.Suaidi, Grayscale MRI Image Compression Using feedforward Neural Networks, Proceedings of the 6th International conference on Broadband Communications \& Biomedical Application, November 21-24,2011.

[10] S.Manimurugan, porkumaran, Secure Medical Image Compression Using Block pixel Sort Algorithm, European Journal of scientific Research, Vol.56 No2 (2011), pp: 129-138.

[11] Jiang.J, Image compression with neural network, A survey. Signal Processing: image communication, 1998.

[12] N.Sriraam, Ahigh-Performance Lossless Compression Scheme for EEG Signals Using Wavelet Transform and Neural network Predictors, International journal of telemedicine and application, Volume 2012.

[13] K Gopi, Dr. T. Rama Shri, Medical Image Compression Using Wavelets, IOSR Journal of VLSI and Signal Processing (IOSR-JVSP) Volume 2, Issue 4 (May. - Jun. 2013), pp:1-6.

[14] G.Vijayvargiya, S.Silakari,R.Pandey,A Novel Medical Image Compression Technique based on Structure Reference Selection using Integer Wavelet Transform Function and PSO Algorithm, International Journal of Computer Applications (0975 - 8887) Volume 91 - No.11, April 2014, pp:9-13.

[15] D.Ravi Varma, "Managing DICOM images: Tips and tricks for the radiologist", Indian J Radiol Imaging. 2012 Jan-Mar; 22(1), pp: 4-1

[16] Tjokorda A.B.W, Adiwijaya,Febri Puguh Permana, Medical Image Watermarking with Tamper Detection and Recovery Using Reversible Watermarking with LSB Modification and Run Length Encoding (RLE) Compression

[17] J. Hyma1, P.V.G.D. Prasad Reddy2, A. Damodaram, Comparative Analysis of Compression Techniques and Feature Extraction for Implementing Medical Image Privacy Using Searchable Encryption, ,Advances in Intelligent Systems and Computing, Springer International Publishing Switzerland 2015, pp:63-69.

[18] A.Younus,G.Raja, A.K.Khan, Hybrid Compression of Medical Images Based on Lapped Biothogonal Transform \& DISCRETE COSINE TRANSFORM 